\title{
Theoretical analysis of strain and strain decay in InAs/GaAs(001) multilayer quantum dot growth
}

\author{
S. Tomića) \\ Computational Science and Engineering Department, CCLRC Daresbury Laboratory, Warrington, \\ Cheshire WA4 4AD, United Kingdom \\ P. Howe ${ }^{\text {b) }}$ \\ Centre for Electronic Materials and Devices, Department of Physics, Imperial College, \\ London SW7 2AZ, United Kingdom
}

\author{
N. M. Harrison \\ Computational Science and Engineering Department, CCLRC Daresbury Laboratory, Warrington, \\ Cheshire WA4 4AD, United Kingdom and Department of Chemistry, Imperial College, \\ London SW7 2AZ, United Kingdom \\ T. S. Jones \\ Centre for Electronic Materials and Devices, Department of Chemistry, Imperial College, \\ London SW7 2AZ, United Kingdom
}

(Received 19 October 2005; accepted 24 February 2006; published online 15 May 2006)

\begin{abstract}
The results of analytically based calculations of the various strain components within and outside InAs quantum dots (QDs) in a GaAs matrix are presented. The calculations performed here take into account cubic crystal strain anisotropy and spatial grading of the indium composition. The assumptions regarding the shape and compositional profile of the QDs have been refined and reflect experimental findings from previous morphological studies. Generally, cone-shaped QDs are modeled with and without truncation, and the composition is either pure InAs or is assumed to change linearly from $50 \%$ at the bottom to $100 \%$ at the top. The exact QD dimensions-height and base diameter-have been obtained from scanning tunneling microscopy and atomic force microscopy. The first part of the calculation addresses structures containing a single QD layer. Particular emphasis is placed on evaluating the decay of strain in the growth direction, as this is known to affect QD nucleation and growth in subsequent layers. In the second part the calculations are expanded to structures containing two layers of QDs with separations of 10, 20, and $30 \mathrm{~nm}$. It is shown that the biaxial strain component decays more rapidly in the case of an isolated QD compared with a QD in the second layer of a structure with $10 \mathrm{~nm}$ spacing. In this bilayer structure, the hydrostatic strain within the first layer QDs is significantly smaller compared with that in the upper QDs and the implications for the electronic band structure are discussed. Our calculations provide insight into trends in (multilayer) QD structures that are not easily observed experimentally. (C) 2006 American Institute of Physics. [DOI: 10.1063/1.2190028]
\end{abstract}

\section{INTRODUCTION}

III-V semiconductor quantum dots (QDs) look promising candidates as the active region in next generation optoelectronic devices, such as low chirp lasers, ${ }_{1}^{1}$ semiconductor optical amplifiers, ${ }^{2,3}$ single photon emitters, ${ }^{4}$ or registers in quantum computing. ${ }^{5}$ The InAs/GaAs(001) QD system is of particular technological interest due to the high optical efficiency that can be realized. Recently, multilayer QD structures have been identified as possible contenders for devices operating at wavelengths greater than $1.3 \mu \mathrm{m} .{ }^{6} \mathrm{QD}$ multilayers are generally employed as the active region for in-plane laser diodes and it is now well established that their growth and properties can be complex, in particular, when the QD layers are separated by relatively small GaAs spacer layers (typically $\leqslant 20 \mathrm{~nm}$ ). In these cases not only the effects of In migration and segregation ${ }^{7,8}$ as well as In/Ga intermixing

\footnotetext{
a) Electronic mail: s.tomic@dl.ac.uk

${ }^{b)}$ Electronic mail: p.howe@imperial.ac.uk
}

during the various growth steps have to be taken into account, ${ }^{6,9-11}$ but also strain interactions. ${ }^{12-15}$ These are important for several reasons: First, strain engineering of QDs is a possible route towards achieving longer wavelength emission for all but the first QD layer by using a templating effect, in which the QDs from the first layer dictate the position of QD nucleation in the second layer and therefore also its QD number density. ${ }^{6,12,16}$ Second, the QDs in the upper layer can, under certain growth conditions, maintain a favorable strain state and compositional profile, which has implications for the electronic band structure and the resulting optical properties. ${ }^{6}$ Third, superposition of strain fields and the resulting build up of strain can be a serious problem when growing several closely spaced QD layers due to the reduction of optical/electrical quality in devices and enhanced surface roughness. ${ }^{17,18}$

This paper uses analytically based strain calculations to evaluate the maximum penetration depth of the strain fields emanating from the QDs into the GaAs cap layer. The cal- 


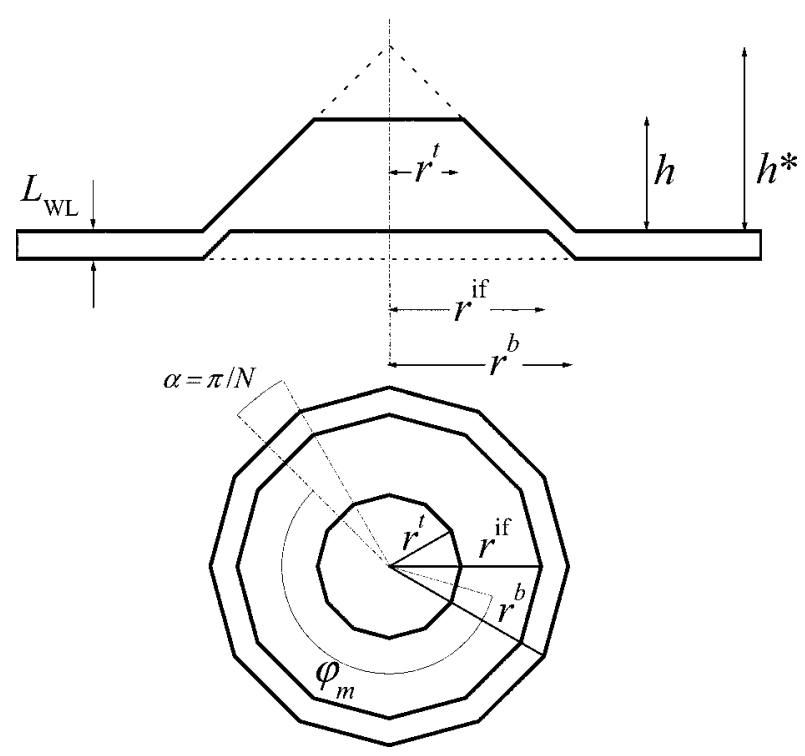

FIG. 1. Schematic of the QD shape, where $h^{*}$ denotes the height of the virtual QD pyramid as stated in Tables I and III, $h$ is the actual height of the $\mathrm{QD}$, and $L_{\mathrm{WL}}$ is the wetting layer thickness (top). The truncation factor is defined as $T=h / h^{*}$. The dotted line underneath the QD represents the profile of the wetting layer omitted in the model. The bottom schematic is a topview representation of the QD shape and defines $\alpha$ and $\varphi_{m}$ (see Appendix). $r^{t}, r^{\text {if }}$, and $r^{b}$ are also defined in the schematics.

culations take into account strain anisotropy of the cubic crystal and vertical grading of the indium composition. Also, the calculations include more realistic assumptions concerning the QD size and shape, factors which are shown to be important in order to correctly assess the behavior of the biaxial strain component and most important its decay in the growth direction. The decay rate has significant implications for the processes of QD nucleation and growth in the subsequent layer, which determine the pairing probabilities. In an experimental study addressing strain decay in InAs/GaAs QD multilayers, our group has recently shown that the templating effect of the first layer QDs does indeed operate very effectively at GaAs spacer layers of $10 \mathrm{~nm}$ and even slightly beyond. ${ }^{16}$ Due to complete decay of the strain fields for GaAs thicknesses of $50 \mathrm{~nm}$ and more, however, QD nucleation and growth in the second InAs layer have been found to be exclusively dictated by the random migration of In adatoms on the surface, which is controlled by the substrate temperature. For intermediate GaAs spacer thicknesses a competition between the two effects has been experimentally observed.

This behavior has been theoretically modeled using an analytical approach based on QDs with a conical shape. Reflecting experimental findings, the composition of the QDs is considered to change linearly from $50 \%\left(\mathrm{In}_{0.5} \mathrm{Ga}_{0.5} \mathrm{As}\right)$ at the bottom to $100 \%$ (InAs) at the top in most of the cases. ${ }^{19}$ Furthermore, the influence of a truncation effect is investigated by varying the truncation factor $(T)$, defined in Fig. 1, from 0.5 to 1 . The presence of a two-dimensional (2D) wetting layer of InAs of thickness of $0.5 \mathrm{~nm}$ is also included in the calculations, for which an anisotropic model of strain $\left(c_{11}-c_{12}-2 c_{14} \neq 0\right)$ has been chosen, reflecting the symmetry of zinc-blende cubic crystals. However, experimental evidence suggests ${ }^{20}$ that at least directly underneath a QD the wetting layer ripens. This has been taken into consideration for the calculations. The details of the calculations are outlined in Sec. II.

In Sec. III A results are presented for an isolated layer of QDs with dimensions reflecting scanning tunneling microscopy (STM) and atomic force microscopy (AFM) measurements of freestanding QDs. During GaAs capping, the QD morphology is known to change, but it is very difficult to extract accurate information about the dimension and shape of buried QDs using techniques such as transmission electron microscopy (TEM), cross-sectional STM, or x-ray diffraction (XRD). ${ }^{20-23}$ The assumed shape is therefore intrinsically unrealistic, but inclusion of the truncation effect in the calculations partly overcomes the problem of overestimating the QD height resulting from measurements of uncapped QDs.

Section III B focuses on bilayer QD structures with separations between the QD layers of 10,20 , and $30 \mathrm{~nm}$, respectively. The decay of strain fields associated with the second layer QDs is known to be different compared with isolated QDs, ${ }^{24}$ because both classes of QDs can exhibit markedly different characteristics. ${ }^{6}$ The QD dimensions for each of the samples with a specific spacer layer thickness are again obtained from STM/AFM measurements and the slightly changed thickness of the wetting layer reflects changes in the critical thickness for QD formation compared with an isolated layer of QDs. ${ }^{25}$ Since the QDs that form the basis of these calculations are formed at a lower substrate temperature, ${ }^{16}$ it can be assumed that both their compositional profile and their final shape correspond much more closely to the ideal case of QDs with a conical shape and an In composition of $100 \%$ throughout the structure. This has been taken into consideration for the calculations presented in Sec. III B.

In previous works pairing probabilities between the first and the second layer QDs have been calculated based on the influence of strain on the surface In adatom diffusion ${ }^{12}$ or based on thermodynamic effects ${ }^{26-28}$ or based on a combination of both thermodynamic and kinetic effects. ${ }^{29,30}$ Recently, various groups have addressed not just aspects concerning the nucleation and growth of the QDs but also the effects of strain and strain interactions on the (opto)electronic properties of mature, capped QDs. ${ }^{31-33}$ The calculations presented in this work will also be utilized as the basis for calculations of the electronic band structure, the details of which will be presented separately.

\section{DETAILS OF THE CALCULATION}

To calculate the strain tensor in an arbitrarily shaped QD and in the surrounding material or different arrangements of the QDs we employ an analytical method developed by O'Reilly and co-workers in Refs. 34-36. The method is based on Green's tensor of the anisotropic elastic medium convoluted with the forces (stress) existing on the surface of an embedded object (in our case the QD) of arbitrary shape. ${ }^{37,38} \mathrm{We}$ outline here the basic formulas and then 
modify them to include grading of the indium composition in an anisotropic elastic medium. The strain tensor component can be expressed in real space as

$$
\varepsilon_{i j}(\mathbf{r})=\frac{(2 \pi)^{3}}{L_{x} L_{y} L_{z}} \sum_{n_{x}, n_{y}, n_{z}} \mathcal{E}_{i j}\left(\boldsymbol{\xi}_{\mathbf{n}}\right) \exp \left(i \boldsymbol{\xi}_{\mathbf{n}} \mathbf{r}\right)
$$

where $L_{x}, L_{y}$, and $L_{z}$ are the dimensions of the supercell in the $x, y$, and $z$ directions, respectively, and $\xi_{\text {n }}$ $=\left(\xi_{x, n_{x}}, \xi_{y, n_{y}}, \xi_{z, n_{z}}\right)$ is the plane wave basis with a particular harmonic along the $\nu$ direction defined as $\xi_{\nu, n_{\nu}}=2 \pi n_{\nu} / L_{\nu}$, with $\nu=(x, y, z) \cdot \mathcal{E}_{i j}\left(\boldsymbol{\xi}_{\mathbf{n}}\right)$ is the Fourier transform of the strain tensor, when strain is expressed in terms of Green's displacement tensor, the elastic moduli of the crystal with cubic symmetry, the stress on the surface of the QD, and the initial displacement due to the different lattice constants between the QD and the matrix material. This Fourier transform can be obtained in analytical form. Under the anisotropic assumption $\left(c_{\text {an }}=c_{11}-c_{12}-2 c_{44} \neq 0\right)$ and equality of the elastic constant in the QD and the surrounding matrix material, this term reads as

$$
\mathcal{E}_{i j}(\boldsymbol{\xi})=\epsilon_{a} \mathcal{X}_{\mathrm{QD}}(\boldsymbol{\xi})\left\{\delta_{i j}-\frac{\left(c_{11}+2 c_{12}\right) \xi_{i} \xi_{j} / \xi^{2}}{1+\left(c_{12}+c_{44}\right) \sum_{\nu}\left[\xi_{\nu}^{2} /\left(c_{44} \xi^{2}+c_{\mathrm{an}} \xi_{\nu}^{2}\right)\right]}\left(\frac{1 / 2}{c_{44}+c_{\mathrm{an}} \xi_{i}^{2} / \xi^{2}}+\frac{1 / 2}{c_{44}+c_{\mathrm{an}} \xi_{j}^{2} / \xi^{2}}\right)\right\}
$$

where $\epsilon_{a}=\left(a_{\mathrm{mx}}-a_{\mathrm{QD}}\right) / a_{\mathrm{QD}}$ is the relative mismatch of the lattice constants of the QD and matrix material and $c_{11}, c_{12}$, and $c_{44}$ are elastic constants for the matrix material. The characteristic function of the truncated pyramid shape with $z$-dependent grading of the indium composition (see Appendix), which enters Eq. (2), is given by

$$
\begin{aligned}
\mathcal{X}_{\mathrm{QD}}(\xi)= & \mathcal{X}_{\mathrm{pyr}}\left(\boldsymbol{\xi}, r^{b}, h^{*}, g^{*}, g^{b}\right)-\exp \left(-i \xi_{z} h\right) \\
& \times \mathcal{X}_{\mathrm{pyr}}\left(\boldsymbol{\xi}, r^{t}, h^{*}-h, g^{*}, g^{t}\right)+\mathcal{X}_{\mathrm{WL}}\left(\xi_{z}, L_{\mathrm{WL}}\right) \\
& -\exp \left(i \xi_{z} L_{\mathrm{WL}}\right) \mathcal{X}_{\mathrm{WL}}^{\mathrm{off}}(\boldsymbol{\xi}),
\end{aligned}
$$

where $r^{b}$ and $r^{t}$ are the radii of the circumscribed circles around the base and top in the $x y$ plane of the pyramid, $h$ is the actual height of the truncated pyramid, $h^{*}=r^{b} h /\left(r^{b}-r^{t}\right)$ is the nominal QD height of the untruncated pyramid, $g^{b}$ is the indium composition at the base of the pyramid (at the WL-QD interface), $g^{t}$ is the indium composition at the top of the QD (set to be 1 in the calculations), and $g^{*}=h^{*}\left(g^{t}\right.$ $\left.-g^{b}\right) / h+g^{b}$ is the indium composition at the virtual apex of the truncated pyramid (see Fig. 1). If the pyramid is untrucated then $g^{*} \equiv g^{t}=1$. $L_{\mathrm{WL}}$ is the wetting layer thickness. $\mathcal{X}_{\mathrm{WL}}^{\mathrm{off}}(\boldsymbol{\xi})$ is the Fourier transform of the wetting layer (WL) removed underneath the QD (Fig. 1) and is defined by

$$
\begin{aligned}
\mathcal{X}_{\mathrm{WL}}^{\mathrm{off}}(\boldsymbol{\xi})= & \mathcal{X}_{\mathrm{pyr}}\left(\boldsymbol{\xi}, r^{b}, h^{*}, 1,1\right)-\exp \left(-i \xi_{z} L_{\mathrm{WL}}\right) \\
& \times \mathcal{X}_{\mathrm{pyr}}\left(\boldsymbol{\xi}, r^{\mathrm{if}}, h^{*}-L_{\mathrm{WL}}, 1,1\right),
\end{aligned}
$$

where $r^{\mathrm{if}}=r^{b}\left(h^{*}-L_{\mathrm{WL}}\right) / h^{*}$ is the radius of the circumscribed circle around the polygon at the WL-QD interface $(z=0)$. The Fourier transform of the wetting layer is given by

$$
\mathcal{X}_{\mathrm{WL}}\left(\xi_{z}, L_{\mathrm{WL}}\right)=\frac{L_{x} L_{y}}{(2 \pi)^{3}} \frac{i}{\xi_{z}}\left[1-\exp \left(i \xi_{z} L_{\mathrm{WL}}\right)\right]
$$

and is assumed to be pure InAs, i.e., no In grading in the WL. For the QD bilayer the characteristic function reads as

$$
\mathcal{X}_{2 \mathrm{QD}}(\boldsymbol{\xi})=\mathcal{X}_{\mathrm{QD}}^{(1)}(\boldsymbol{\xi})+\exp \left(-i \xi_{z} s\right) \mathcal{X}_{\mathrm{QD}}^{(2)}(\boldsymbol{\xi}),
$$

where $\mathcal{X}_{\mathrm{QD}}^{(i)}(\xi)$ are characteristic functions of the individual dots (not necessarily identical) in layer $i$ given by Eq. (3), and $s$ is the spacer layer thickness or vertical distance between the layers.

\section{RESULTS AND DISCUSSION}

\section{A. Isolated QD layer}

The exact assumptions regarding the QD and WL characteristics, on which the strain calculations for single QD layers are based, are summarized in Table I. The calculations were carried out on a cuboid-shaped supercell with a thickness of $50 \mathrm{~nm}$ in both the $x$ and $y$ directions and $70 \mathrm{~nm}$ in the $z$ direction. The interface between the GaAs matrix and the $\mathrm{WL}$ is located at $z=0$. A schematic of the WL and QD system is shown in Fig. 1. The truncation factor is defined as $T$ $=h / h^{*}$, where $h^{*}$ and $h$ are the nominal and real heights of a truncated pyramid respectively, measured from the top of the WL. To model the conical shape of the QDs we assume the number of pyramid sides to be $N=60$, which corresponds to an angle of $\alpha=3^{\circ}$ for each individual segment. Due to experimental evidence of a ripening effect that this InAs directly underneath the QD experiences, ${ }^{20}$ the calculations

TABLE I. Assumptions for the $T$ factor (truncation factor) as defined in the main text (Sec. III A), the nominal QD height $\left(h^{*}\right)$, and QD base diameter $\left(r^{b}\right)$. In all cases the thickness of the wetting layer was fixed at $0.5 \mathrm{~nm}$ and the In composition changes linearly from $50 \%$ at the bottom to $100 \%$ at the top of the QDs. The wetting layer was assumed to exhibit the characteristics shown schematically in Fig. 1.

\begin{tabular}{clll}
\hline \hline Sample & $T$ factor & $h^{*}(\mathrm{~nm})$ & $r^{b}(\mathrm{~nm})$ \\
\hline 1 & 1 & 5.9 & 22 \\
2 & 0.75 & 5.9 & 22 \\
3 & 0.5 & 5.9 & 22 \\
4 & 0.75 & 7.38 & 22 \\
5 & 0.75 & 5.9 & 24.6 \\
\hline
\end{tabular}



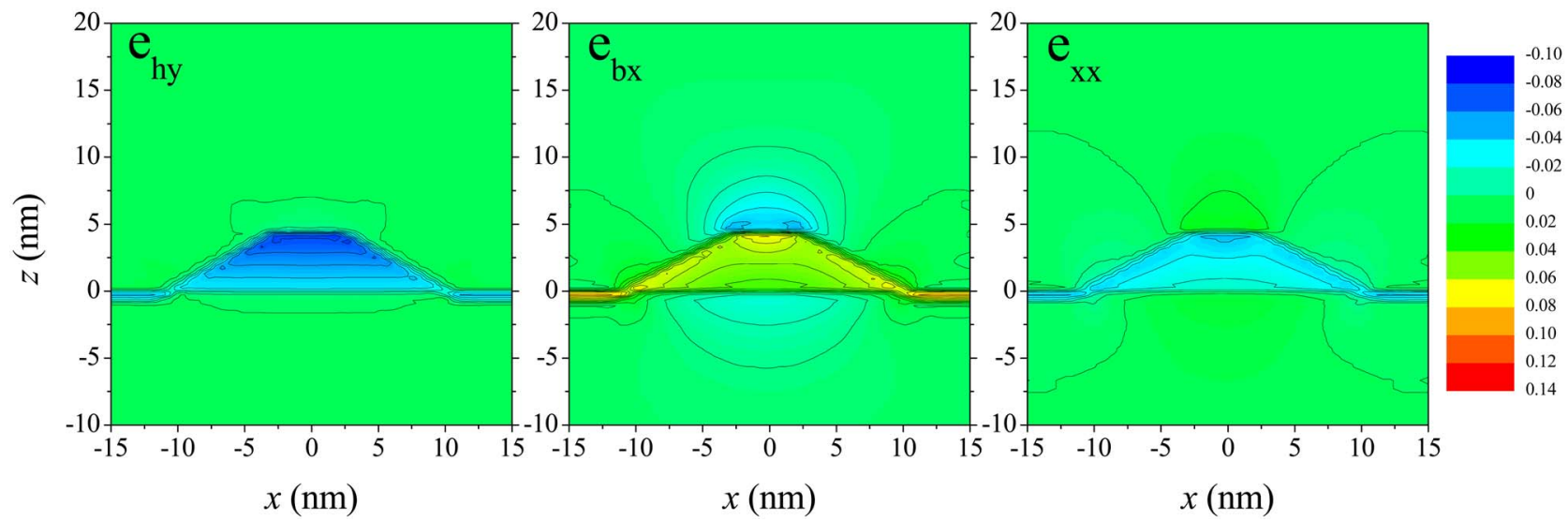

FIG. 2. (Color online) Graphical representation of the three strain components (hydrostatic strain, biaxial strain, and $\varepsilon_{x x}$ ) in the $x z$ plane through $y=0$ of the QD from sample 2. Contour lines are also shown as a guide.

have been carried out assuming the WL geometry shown in Fig. 1. Without this omission, the strain profiles would be unrealistic (abrupt WL/QD interface) if the WL was assumed to be pure InAs. It should be emphasized that omission of the WL does not lead to a measurable effect of strain inside the QDs as well as in the GaAs matrix above.

As relevant components for probing the strain state of the system of the QD and its environment, the biaxial strain $\left[\varepsilon_{\mathrm{bx}}=\varepsilon_{z z}-\left(\varepsilon_{x x}+\varepsilon_{y y}\right) / 2\right]$, hydrostatic strain $\left(\varepsilon_{\mathrm{hy}}=\varepsilon_{z z}+\varepsilon_{x x}\right.$ $\left.+\varepsilon_{y y}\right)$, and the strain component in the $x$ direction $\left(\varepsilon_{x x}\right)$ will be presented. Shear stresses will not be discussed. The QDs are assumed to be completely buried within the GaAs and surface effects can be neglected.

Figure 2 shows maps of the hydrostatic strain, biaxial strain, and strain component $\varepsilon_{x x}$ in the (101) plane ( $x z$ plane) through $y=0$ for the QD of sample 2. Grading of the composition, assumed for all isolated dots, as well as the QD shape are clearly visible. The hydrostatic strain is mostly confined within the QD and WL and it is practically zero immediately outside the QD side edges. The only detectable effects in the GaAs matrix are located directly above and underneath the QD. The hydrostatic strain profile and overall magnitude within the QD have important implications for their electronic band structure when fully enclosed in the GaAs matrix. The $\varepsilon_{x x}$ component is also relatively weak, but has a slightly greater penetration depth compared with the hydrostatic strain. The main areas under strain are located directly above the QD as well as outside and underneath the side edges.

By contrast, the biaxial strain within the QDs is positive, while outside it is negative. The largest gradients of the respective strain components are located at the top InAs/GaAs interface, which is the reason for significant material intermixing and redistribution at the top of the QDs during capping with GaAs. The effects of material intermixing have been experimentally observed by cross-sectional TEM studies of fully capped QDs, which clearly show that the initially formed QD apex, as measured by STM, disappears during overgrowth with GaAs and the resulting compositional profile resembles more a half lens or a truncated cone with
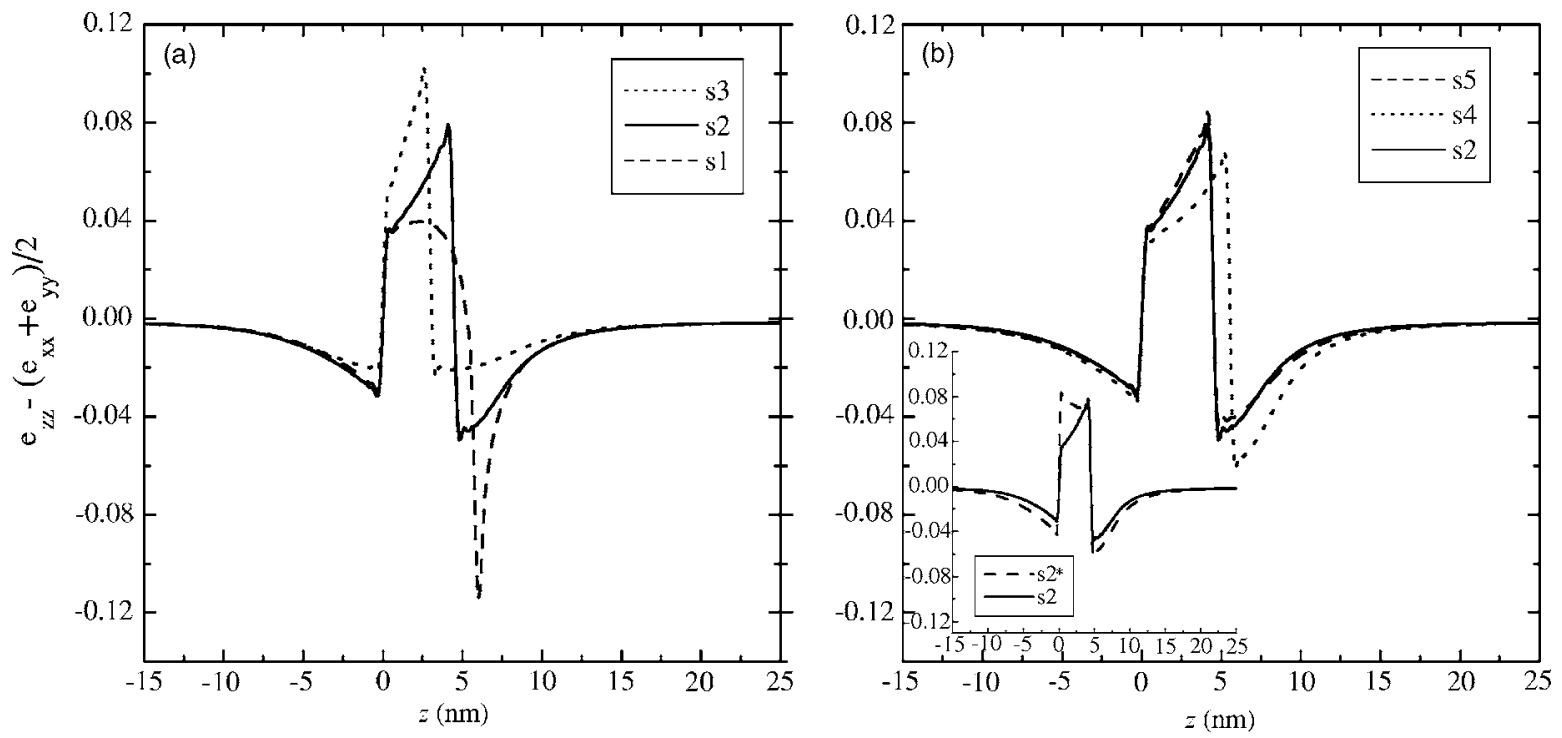

FIG. 3. Profiles of the biaxial strain component in the $z$ direction [001], through the QD midpoint $(x=y=0)$ for samples $1-3$ (a) and samples 2, 4, and 5 (b). Inset: comparison of the strain decay in the single QD layer, sample $2^{*}$, having no indium composition grading (dashed line) with sample 2 (solid line), in which indium composition is graded from $50 \%$ at the bottom to $100 \%$ at the top. 
TABLE II. Thickness of the GaAs above the QD, at which the calculated In diffusion coefficients are reduced to $90 \%$ and $80 \%$, respectively, of the value on unstrained $c(4 \times 4)$ GaAs. The calculations are based on Ref. 40 .

\begin{tabular}{clcc}
\hline \hline Sample & $T$ factor & $z_{d}^{90 \%}(\mathrm{~nm})$ & $z_{d}^{80 \%}(\mathrm{~nm})$ \\
\hline 1 & 1 & 15.6 & 11.8 \\
2 & 0.75 & 16.2 & 11.9 \\
$2^{*}$ & 0.75 & 18.3 & 13.5 \\
3 & 0.5 & 15.2 & 10.9 \\
4 & 0.75 & 17.7 & 13.4 \\
5 & 0.75 & 17.1 & 12.6 \\
\hline \hline
\end{tabular}

smooth edges. ${ }^{21,39}$ In particular, the (top) InAs/GaAs interfaces, both of the WL as well as of the QDs, are known to be far from atomically sharp, which is due to a combination of lattice mismatch and the differences in bond strength between InAs and GaAs. ${ }^{7}$

The magnitude of strain and the strain gradients are particularly high at the tip of a nontruncated cone (e.g., $T$ factor $=1$; sample 1 not shown), but it should be emphasized that this assumption is highly unrealistic for a buried QD as strain-driven intermixing will occur during capping and reduce the stress. A more quantitative analysis of the strain profile is necessary for a thorough understanding. Figure 3(a) shows profiles of the biaxial strain components through the QD center in the [001] direction for samples 1-3. The only difference in these samples is the truncation, which is obvious when comparing the thicknesses of the regions under compressive strain in the plots. The shape of the profile is qualitatively very similar in samples 2 and 3 , but sample 1 exhibits several interesting differences. The following discussion will focus on the strain profiles within the QDs (for sample $1 \mathrm{a} z$ value between 0 and $5.9 \mathrm{~nm}$, for sample 2 a $z$ value between 0 and $4.475 \mathrm{~nm}$, and for sample 3 a $z$ value between 0 and $2.95 \mathrm{~nm}$ ). The biaxial strain rises linearly in samples 2 and 3 from $z=0 \mathrm{~nm}$ up to the InAs/GaAs interface. From there it decreases in a discontinuous manner to its overall minimum (indicating a large strain gradient) from where it rises slowly to eventually reach zero. In contrast, the strain within QD sample 1 does not rise linearly, but describes a curve with a maximum positive value $\sim 0.04$ around $z=2 \mathrm{~nm}$; from there it falls sharply to $\sim-0.117$ at $z$ $=5.9 \mathrm{~nm}$. Interestingly, the strain reaches zero at just over $5 \mathrm{~nm}$, indicating that the tip of the QD is under tensile strain.

The areas above the QD/GaAs interface are under tensile strain for all samples. The effect of an increased lattice con- stant within the GaAs matrix has been used to explain QD vertical self-alignment in multilayer structures. ${ }^{12,40}$ The emphasis of the calculations presented here is to quantify the penetration depth of the strain fields and to study the effects of variations in QD shape, size, and composition on this penetration depth. In order to accomplish this it is necessary to choose a cutoff value, which is the magnitude of strain below which In adatom migration on a GaAs layer above a buried QD layer starts to be affected. For thicker GaAs layers no interaction between the QD layers should be detectable. Choosing an appropriate cutoff value for the tensile strain in the GaAs matrix requires consideration of kinetic effects, such as In adatom diffusion. However, even if a direct correlation between strain and surface In diffusion is established, it is not straightforward to choose a threshold for the strain, because the transition regime between fully correlated QD growth and noncorrelated growth will be relatively large. In a recent work by Penev et al., based on ab initio density-functional theory, the diffusion coefficient $D(\varepsilon)$ for In adatoms on a $c(4 \times 4)$ reconstructed $\mathrm{GaAs}(001)$ surface has been calculated by taking into account the tensile strain emanating from a buried QD. ${ }^{40}$ Based on their model, the reduction in $D(\varepsilon)$ for $\varepsilon=-0.3 \%$ would be approximately $9 \%$ at $475{ }^{\circ} \mathrm{C}$. We have determined the $z$ values above the various QDs at which the reductions in $D(\varepsilon)$ were $10 \%$ and $20 \%$, respectively $\left(z_{d}^{90 \%}\right.$ and $z_{d}^{80 \%}$, Table II). These values can be arbitrarily regarded as the before-mentioned cutoff values (even though a thorough analysis would have to be carried out to include a full model of surface In adatom diffusion in order to determine whether this local reduction in diffusion coefficient is enough to force QD nucleation). A reduction of $10 \%(20 \%)$ in the In diffusion coefficient is reached with a biaxial strain of $-0.0033(-0.0075)$.

Based on Fig. 3(a) it is clear that the penetration depth of the strain varies slightly between samples 1-3 and the results presented in Table II confirm this. However, comparing the latter with experimentally determined values ${ }^{16}$ suggests that the calculations either underestimate the magnitude of strain in the GaAs or the chosen threshold values for the In diffusion coefficient are not appropriate. Previous experimental work suggests that strain effects are significant up to and above $30 \mathrm{~nm} .^{16,24}$

The inset in Fig. 3(b) shows a comparison between sample 2 and a reference sample $2^{*}$ with QDs of pure InAs.

TABLE III. Assumptions for the $T$ factor and WL thickness $\left(L_{\mathrm{WL}}\right)$, In composition profile from QD bottom to top, nominal QD height $\left(h_{2 \text { nd }}^{*}\right)$, and QD base diameter of the second layer QDs $\left(r_{2 \text { d d }}^{b}\right)$. The characteristics of the QDs from the first layer are the same as those of sample 2 (see Table I) and the spacings between the layers is $10 \mathrm{~nm}$ (samples 6-8), $20 \mathrm{~nm}$ (sample 9), and $30 \mathrm{~nm}$ (sample 10). In all cases the WL is omitted underneath the QDs, as shown in Fig. 1.

\begin{tabular}{cccccc}
\hline \hline Sample & $T$ factor & $L_{\mathrm{WL}}(\mathrm{nm})$ & In profile & $h_{\text {2nd }}^{*}(\mathrm{~nm})$ & $r_{\text {2nd }}^{b}(\mathrm{~nm})$ \\
\hline 6 & 0.75 & 0.4 & 1 & 6.96 & 27.8 \\
7 & 0.75 & 0.4 & $0.5 \rightarrow 1$ & 6.96 & 27.8 \\
8 & 1 & 0.4 & $0.5 \rightarrow 1$ & 6.96 & 27.8 \\
9 & 0.75 & 0.45 & $0.5 \rightarrow 1$ & 6.52 & 28.98 \\
10 & 0.75 & 0.5 & $0.5 \rightarrow 1$ & 3.63 & 23.53 \\
\hline \hline
\end{tabular}



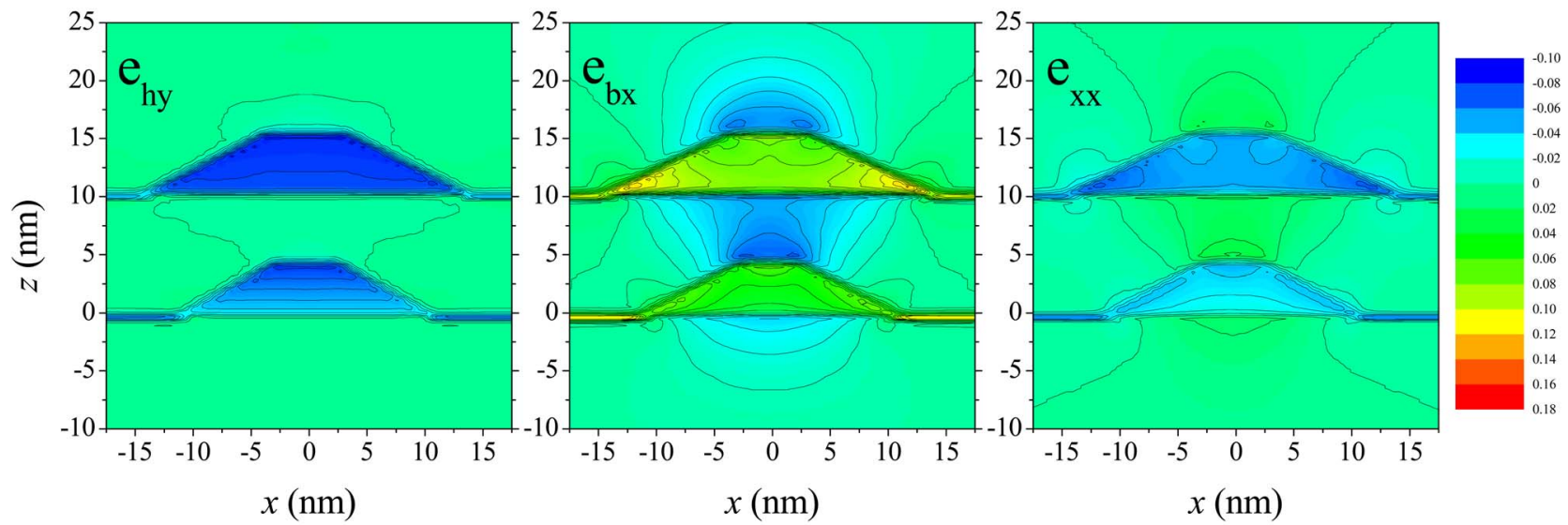

FIG. 4. (Color online) Maps of the three strain components (hydrostatic strain, biaxial strain, and $\varepsilon_{x x}$ ) in the $x z$ plane through the center of the QD from sample 6. Contour lines are also shown as a guide.

The difference in $z_{d}^{90 \%}$ and $z_{d}^{80 \%}$ between the two samples is between 1.6 and $2.1 \mathrm{~nm}$ and is solely due to the different $\mathrm{In}$ composition (see Table II).

In the following the emphasis will be on two particular cases of QDs, although similar to sample 1 the assumptions for the QD geometry are unrealistic. In samples 4 and 5 the total QD volume was increased by 25\% compared with sample 2. In the first case this was accomplished by maintaining the QD base diameter at $22 \mathrm{~nm}$ and increasing the nominal QD height to $7.38 \mathrm{~nm}$, whereas in sample 5 the increase in volume was due solely to an increase in base diameter (to $24.6 \mathrm{~nm}$ ). This series of samples gives an indication of whether the total QD height, base diameter, or volume determines the magnitude of strain. Figure 3(b) shows profiles of the biaxial strain in samples 4 and 5 as well as sample 2 . The similarity of the profiles for samples 2 and 5 indicates that the increase in base diameter only leads to a very small change in strain profile. The strain profile in sample 4, however, is very different, with less strain within the QD and a pronounced increase in strain in the GaAs layer above. The $z_{d}^{80 \%}$ and $z_{d}^{90 \%}$ values (see Table II) from sample 4 are $1.5 \mathrm{~nm}$ larger than those calculated from sample 2, implying that the strain propagates $1.5 \mathrm{~nm}$ further into the cap- ping layer. It is interesting to note that the difference in real QD height between the two samples is $\sim 1.1 \mathrm{~nm}$, suggesting that an incremental change in height increases the maximum strain penetration by a little more than this amount. However, it is worth emphasizing that this observation should be treated with caution since it is only true for one particular set of samples. From the previous work of Andreev et al. it is known that the stress outside the QD is essentially independent of its shape. ${ }^{34}$ Based on the comparison between samples 2 and 5 we can confirm that the differences in the $z_{d}^{80 \%}$ and $z_{d}^{90 \%}$ values are small (between 0.7 and $0.9 \mathrm{~nm}$ ) despite the change in QD diameter $(2.6 \mathrm{~nm})$. This highlights the difficulties when trying to extract information about the exact shape of buried QDs by using the results of analytical calculations.

The large values for the strain gradients at the various GaAs/InAs interfaces (seen in the discontinuities of the strain profile) do not reflect the true state of the fully encapsulated dots, because strain-driven intermixing will occur during encapsulation. The resulting "real" strain profiles will be far less discontinuous as suggested by the results of the calculations, but further away from the interfaces (e.g., the
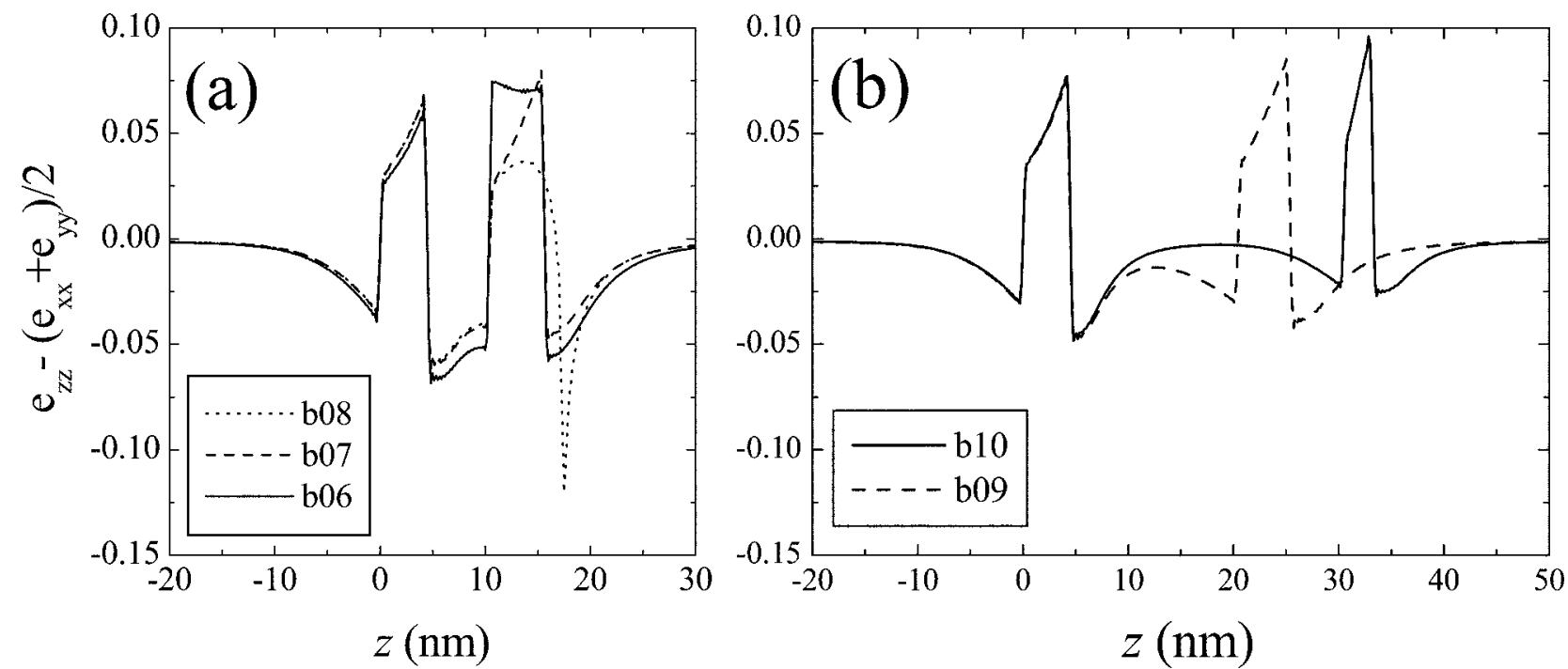

FIG. 5. Profiles of the biaxial strain component in the $z$ direction through the QD midpoint for samples 6-8 (a) and for samples 9 and 10 (b). 
TABLE IV. Thickness of the GaAs above the second QD at which the calculated In diffusion coefficients are reduced to $90 \%$ and $80 \%$, respectively, of the value of unstrained $c(4 \times 4) \mathrm{GaAs}$.

\begin{tabular}{ccccc}
\hline \hline Sample & $T$ factor & Spacer $(\mathrm{nm})$ & $z_{d}^{90 \%}$ & $z_{d}^{80 \%}(\mathrm{~nm})$ \\
\hline 6 & 0.75 & 10 & 21.7 & 16.5 \\
7 & 0.75 & 10 & 19.5 & 14.8 \\
8 & 1 & 10 & 19.4 & 14.8 \\
9 & 0.75 & 20 & 19.0 & 14.1 \\
10 & 0.75 & 30 & 12.4 & 8.9 \\
\hline \hline
\end{tabular}

strain fields in the GaAs or in the center of the QDs) the calculations do provide a more realistic reflection of the system.

\section{B. Quantum dot bilayer}

The assumptions for the strain calculations of the QD bilayer structures are presented in Table III. In all cases the characteristics of the lower QD layer are based on sample 2. In order to accommodate the increased size of the system in the $z$ direction, the supercell dimensions have been extended to between 100 and $120 \mathrm{~nm}$ depending on the thickness of the GaAs spacer layer. In the following the discussion will be restricted to the upper (second) QD layer in all bilayer samples.

Figure 4 shows maps of the hydrostatic, biaxial, and unilateral strains in the (101) plane ( $x z$ plane) through $y=0$ of the QD for sample 6. The description is qualitatively similar to the case of a single QD (sample 2) and the same observations regarding the respective strain components can be made. It should be emphasized again that the upper QD is pure InAs (no compositional grading) and its dimensions are increased with respect to the first QD layer, in agreement with experimental findings. ${ }^{16}$

For a more quantitative understanding of the bilayer system the biaxial strain component through the QD center in the [001] direction is plotted in Fig. 5(a) (samples 6-8) and Fig. 5(b) (samples 9 and 10). Based on our previous discussion the qualitative shapes of the plots are obvious. This includes (i) grading of the In composition in the second QD layer in samples 7-10 with a linear increase in strain profile, (ii) a $T$ factor of 1 for the upper QD in sample 8, and (iii) spacer layers of 20 and $30 \mathrm{~nm}$ for samples 9 and 10, respectively, as opposed to $10 \mathrm{~nm}$ for samples $6-8$.
In the following the various contributions to the differences in strain between pairs of samples will be assessed and are presented in Table V. The strain profiles for samples 7 and 8 are visually indistinguishable from approximately $z$ $=22 \mathrm{~nm}$ onwards. Considering the total thickness of the GaAs spacer layer and the second WL $(10.4 \mathrm{~nm})$ this corresponds to a thickness of the second GaAs layer of $11.6 \mathrm{~nm}$. In other words, after deposition of $\sim 11.6 \mathrm{~nm}$ of GaAs in samples 7 and 8 , there is no visual difference in strain. A more detailed investigation based on the exact values of the biaxial strain and its calculated effect on the In adatom diffusion coefficient has again been carried out and the results are presented in Table IV. It shows that the $z_{d}^{80 \%}$ and $z_{d}^{90 \%}$ values are practically identical for the two samples. The values calculated for sample 6 show that the strain reaches approximately $1.7-2.2 \mathrm{~nm}$ further, which is solely due to the higher In content in the QDs. This is very similar to the difference between the isolated QDs from samples 2 and $2^{*}$ $(1.6-2.1 \mathrm{~nm})$, suggesting that the In profile has similar effects in isolated and stacked QDs. Table V summarizes the effects of variations in the QD dimensions and compositions with regard to the observed changes in $z_{d}^{80 \%}$ and $z_{d}^{90 \%}$ between selected pairs of samples.

Direct comparison of the $z_{d}^{80 \%}$ and $z_{d}^{90 \%}$ values for samples 2 and 7 (same $T$ factor and compositional profile for both) shows that the biaxial strain component reaches between 2.9 and $3.3 \mathrm{~nm}$ further in the upper QD layer (see Table V). The increased QD height for sample $7(\Delta h$ $=0.8 \mathrm{~nm}$ ) can again explain part of this, but the change in QD base diameter must also be considered. Its contribution is more difficult to assess. Based on the results from the previous section a value of approximately $1.8 \mathrm{~nm}$ can be extracted. The remaining $0.3-0.7 \mathrm{~nm}$ can be rationalized in terms of the influence of the first QD layer and the resulting superposition of strain fields.

Comparison between the $z_{d}^{80 \%}$ and $z_{d}^{90 \%}$ values for samples 2 and 6 (same $T$ factor, but different compositional profiles) yields an even more dramatic difference of between 4.6 and $5.5 \mathrm{~nm}$, respectively (see Table V). Comparing these two samples is very important, because the assumptions for the respective QD characteristics are probably the most realistic from the set of samples under discussion. ${ }^{6,16,19}$ Again, the difference in real QD height between the two samples $(\Delta h=0.8 \mathrm{~nm})$, the difference in base diameter, and the resulting contribution of $\sim 1.8 \mathrm{~nm}$ combined with the different $\mathrm{In}$

TABLE V. Comparison of the differences in $z^{80 \%}$ and $z^{90 \%}$ values between selected pairs of samples with the various contributions responsible for these differences (QD composition, QD height, QD diameter, and the effect from strain superposition).

\begin{tabular}{ccccccc}
\hline \hline Samples & $\Delta z_{d}^{90 \%}(\mathrm{~nm})$ & $\Delta z_{d}^{80 \%}(\mathrm{~nm})$ & $\Delta$ comp $(\mathrm{nm})$ & $\Delta h(\mathrm{~nm})$ & $\Delta d(\mathrm{~nm})$ & Superposition \\
\hline $2 / 2^{*}$ & 2.1 & 1.6 & $1.6-2.1$ & 0 & 0 & $\ldots$ \\
$2 / 4$ & 1.5 & 1.5 & 0 & 1.1 & 0 & $\ldots$ \\
$2 / 5$ & 0.9 & 0.7 & 0 & 0 & $\ldots$ & $\ldots$ \\
$6 / 7$ & 2.2 & 1.7 & $1.7-2.2$ & 0 & 0 & $\ldots$ \\
$2 / 7$ & 3.3 & 2.9 & 0 & 0.8 & 1.8 & $0.3-0.7$ \\
$2 / 6$ & 5.5 & 4.6 & $1.7-2.2$ & 0.8 & 1.8 & $0.3-0.7$ \\
$2 / 9$ & 2.8 & 2.2 & 0 & 0.6 & 2.1 & 0 \\
\hline \hline
\end{tabular}



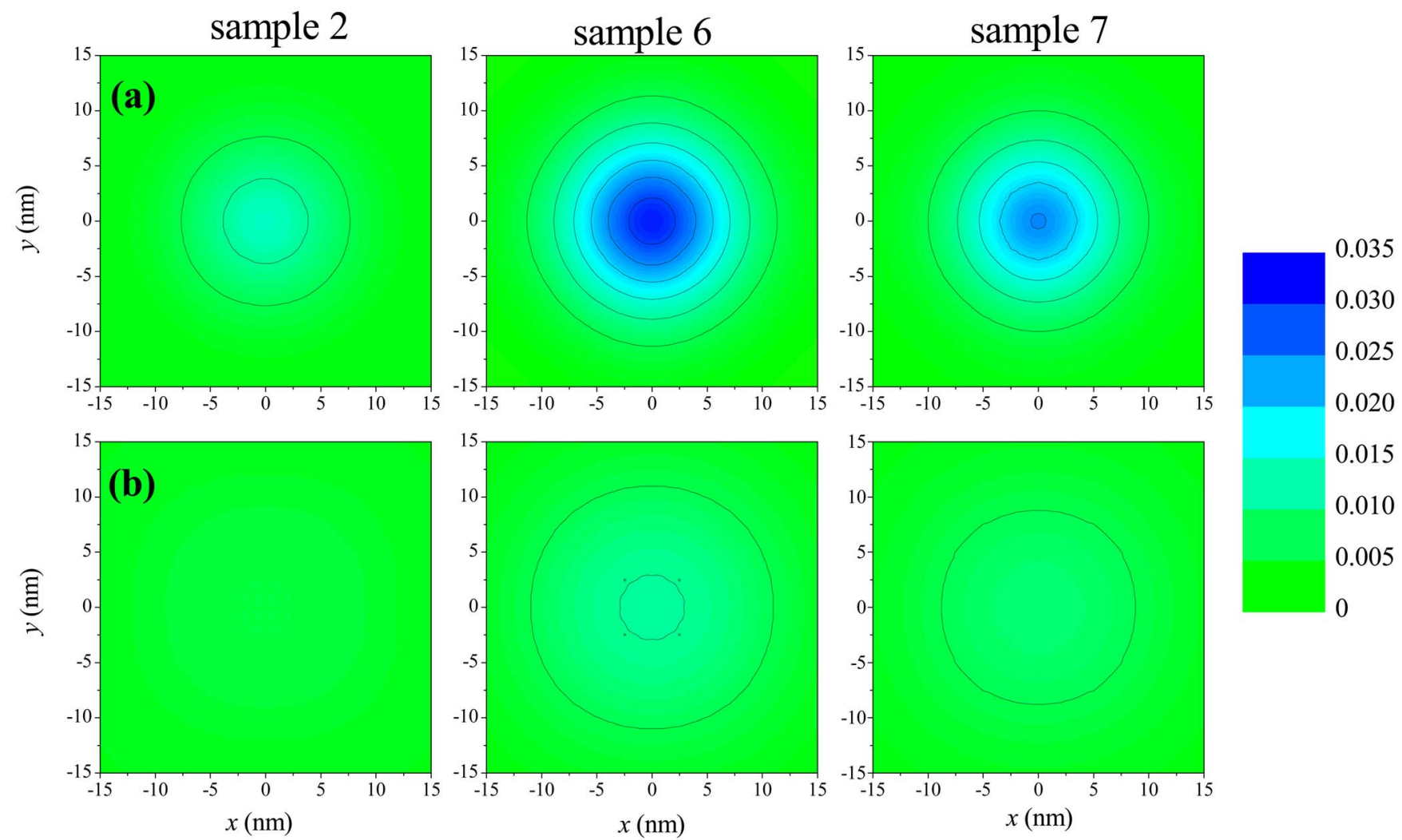

FIG. 6. (Color online) Maps of the biaxial strain components in the $x y$ plane $10 \mathrm{~nm}$ (a) and $15 \mathrm{~nm}$ (6) above the upper QDs (samples 2, 6, and 7). Contour lines are also shown as a guide.

compositions, which account for approximately 1.7/2.2 nm (see comparison 6/7), cannot fully explain these increases in $z_{d}^{80 \%}$ and $z_{d}^{90 \%}$. It must therefore be a combination of $\Delta h, \Delta r^{b}$, the difference in In composition, and the superposition of strain fields from both QDs, the latter contributing again approximately $0.3-0.7 \mathrm{~nm}$. For completeness the biaxial strain components in the $x y$ plane 10 and $15 \mathrm{~nm}$ above the QDs from samples 2, 6, and 7, respectively, are shown in Fig. 6.

If a comparison between the $z_{d}^{80 \%}$ and $z_{d}^{90 \%}$ values of samples 2 and 9 is conducted (same composition and $T$ factor, but different heights and diameters), the respective contributions of differences in QD height $(0.6 \mathrm{~nm})$ and QD diameter $(\sim 2.1 \mathrm{~nm})$ can fully explain the differences and no evidence for superposition of strain fields exists (Table V).
These investigations show that the penetration depth of the strain fields can indeed be quantified and attributed to (i) the QD characteristics (height, base diameter, $T$ factor, and In composition), and (ii) the presence or absence of strain fields from an underlying first QD layer. The vertical superposition of strain fields generally has a small effect and is probably not detectable for spacer layers well in excess of $10 \mathrm{~nm}$. An accurate knowledge of the QD dimensions and their compositional profile is therefore a prerequisite for predicting the magnitude of the strain decay.

So far the discussion has focused on analyzing the biaxial strain above and within the QDs. In order to assess the electronic properties of the QDs, however, consideration of the hydrostatic strain distribution within the QDs is para-
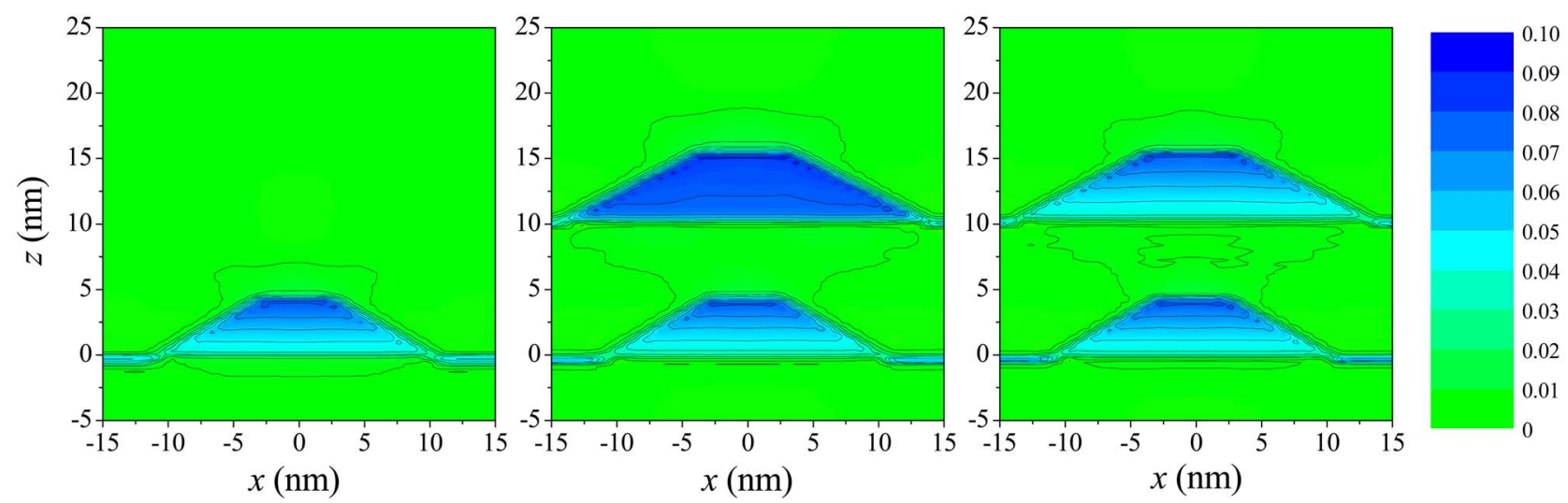

FIG. 7. (Color online) Maps of the hydrostatic strain component in the $x z$ plane through the center of the QDs from samples 2, 6, and 7. Contour lines are also shown as a guide. 
mount, as it too leads to changes in the electronic band structure. Figure 7 shows the hydrostatic strain component in the $x z$ plane through the center of the QDs from samples 2, 6, and 7. The lowest value of hydrostatic strain found for sample 2 reflects the trends already discussed. Furthermore, a detectable difference between samples 6 and 7 should translate into a small variation in the electronic band structure and, ultimately, to a difference in optical emission wavelength. These preliminary results show how our analytical approach can be used in conjunction with experimentally determined QD characteristics to predict optical properties. However, a thorough band calculation taking the strain state of the QDs into account is beyond the scope of this work and will be presented later.

\section{CONCLUSIONS}

The results presented here generally show that the choice of assumptions regarding the QD characteristics is important in order to correctly estimate the strain state within and outside (e.g., above) InAs/GaAs QDs in both single and bilayer structures. In some cases unrealistic assumptions have been made in order to investigate trends and it is also shown that material intermixing must occur at the QD apex during GaAs encapsulation. The biaxial strain component qualitatively follows the compositional profile within the QDs unless the assumptions are unrealistic. In order to quantify the strain decay, the magnitude of biaxial strain at which the In adatom mobility is reduced to just $80 \%$ and $90 \%$, respectively, is calculated (based on Ref. 40) and the height above the QDs at which this strain is reached (the $z_{d}^{80 \%}$ and $z_{d}^{90 \%}$ values) determined. A comparison between different pairs of samples shows that (i) a change in QD height by a certain amount leads to an increase in $z_{d}^{80 \%}$ and $z_{d}^{90 \%}$ by a little more than this change, (ii) a change in QD base diameter by $1 \mathrm{~nm}$ increases the $z_{d}^{80 \%}$ and $z_{d}^{90 \%}$ values by $\sim 1 / 3 \mathrm{~nm}$, (iii) changes in the compositional profile have a pronounced effect on the decay of strain, but the effect of truncation is ambiguous, and (iv) the superposition of strain fields between two vertically aligned QDs can also have a small effect, but not for spacer layer thicknesses of significantly more than $10 \mathrm{~nm}$. Generally, the biaxial strain is found to propagate vertically up to $\sim 16.2 \mathrm{~nm}$ through the GaAs matrix in the case of a realistic single QD, thus giving rise to the possibility of vertical QD alignment. In a bilayer structure with a spacer layer of $10 \mathrm{~nm}$ and equally realistic assumptions regarding the QD characteristics, the penetration depth of the strain is found to be increased by $5.5 \mathrm{~nm}$. This increase is a combination of in- creased In composition and dimensions of the QDs in the second layer and the superposition of strain. However, compared with experimentally observed strain penetration depths (up to $40 \mathrm{~nm}$ for isolated QD layers and $50 \mathrm{~nm}$ for QD bilayers), these values are still too small, implying that the calculations underestimate the strain outside the QDs.

\section{ACKNOWLEDGMENTS}

The authors are grateful to the Engineering and Physical Sciences Research Council (UK) and the CCP3 Consortium for supporting this work. The authors to thank I. J. Bush, B. G. Searle, A. G. Sunderland, and M. Tadić for useful discussions.

\section{APPENDIX}

The characteristic function, $\mathcal{X}_{\mathrm{pyr}}$, of an $N$-sided pyramid can be expressed as a superposition of pyramid segments,

$$
\mathcal{X}_{\mathrm{pyr}}\left(\boldsymbol{\xi}, r, h, g_{u}, g_{d}\right)=\sum_{m=0}^{N-1} \mathcal{X}_{\mathrm{seg}}\left(\boldsymbol{\zeta}_{m}, r, h, g_{u}, g_{d}\right),
$$

each of which is rotated by an angle $\varphi_{m}=2 m \pi / N$ around the vertical axis. The rotated basis set of the plane waves for each segment reads as

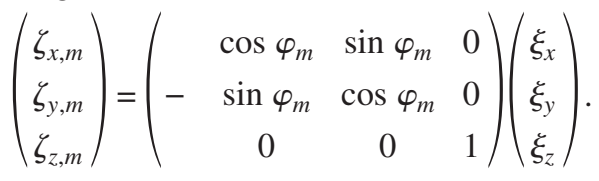

In order to introduce the effect of the spatial grading of the indium composition throughout the QD, i.e., spatial distribution of the strain relative to the mismatch of the dot and matrix material lattice constants $\epsilon_{a}$, the characteristic function of the QD or its segment should be modified to

$$
\mathcal{X}_{\text {seg }}=\frac{1}{(2 \pi)^{3}} \int_{\Omega} g(\mathbf{r}) \exp (-i \boldsymbol{\xi} \mathbf{r}) d \mathbf{r},
$$

where $g(\mathbf{r})$ is the spatial variation of the indium composition $\left(\mathrm{In}_{x} \mathrm{Ga}_{1-x} \mathrm{As}\right)$ inside the $\mathrm{QD}$ relative to the InAs. The remaining task is to model the spatial grading along the vertical direction. In this special case the grading function reduces to $g(\mathbf{r}) \equiv g(z)$. Assuming linear variation of the In concentration from $g_{d}$ at $z=0$ up to $g_{u}$ at the top of the pyramid $(z=h)$, the grading function becomes $g(z)=\left(g_{u}-g_{d}\right)(z / h)+g_{d}$. After introducing the grading function $g(z)$ in Eq. (A3) and integrating over the pyramid segment volume $d \mathbf{r}$, the analytical solution can be expressed in terms of the sum of integral functions:

$$
\begin{aligned}
\mathcal{X}_{\mathrm{seg}}\left(\zeta, r, h, g_{u}, g_{d}\right)= & -\frac{1}{(2 \pi)^{3}} \frac{1}{\zeta_{y} \zeta_{z}}\left[+I_{0}\left(b, \kappa_{1}\right) e^{-i \zeta_{z} h}\left(g_{u}+\frac{g_{u}-g_{d}}{i \zeta_{z} h}\right)-I_{1}\left(b, \kappa_{1}\right) e^{-i \zeta_{z} h}\left(\frac{g_{u}-g_{d}}{b}\right)-I_{0}\left(b, \kappa_{2}\right)\left(\frac{g_{u}-g_{d}}{i \zeta_{z} h}+g_{d}\right)\right. \\
& \left.-I_{0}\left(b, \kappa_{3}\right) e^{-i \zeta_{z} h}\left(g_{u}+\frac{g_{u}-g_{d}}{i \zeta_{z} h}\right)+I_{1}\left(b, \kappa_{3}\right) e^{-i \zeta_{z} h}\left(\frac{g_{u}-g_{d}}{b}\right)+I_{0}\left(b, \kappa_{4}\right)\left(\frac{g_{u}-g_{d}}{i \zeta_{z} h}+g_{d}\right)\right],
\end{aligned}
$$


where $b=r \cos \alpha$ is the horizontal distance along the median from the pyramid center to its segment; $\alpha=\pi / N$ is the half angle of the segment in the $x y$ plane, and

$$
\begin{aligned}
& \kappa_{1}=-\zeta_{x}-\zeta_{y} \tan \alpha+\zeta_{z} h / b, \\
& \kappa_{2}=-\zeta_{x}-\zeta_{y} \tan \alpha, \\
& \kappa_{3}=-\zeta_{x}+\zeta_{y} \tan \alpha+\zeta_{z} h / b, \\
& \kappa_{4}=-\zeta_{x}+\zeta_{y} \tan \alpha .
\end{aligned}
$$

The integral function in Eq. (A4) is given with the recurrent relation $^{41}$

$$
I_{m}(a, \kappa)=\int_{0}^{a} x^{m} e^{i \kappa x} d x=\frac{a^{m}}{i \kappa} e^{i \kappa a}-\frac{m}{i \kappa} I_{m-1}(a, \kappa),
$$

where $I_{0}=\left(e^{i \kappa a}-1\right) /(i \kappa)$ and can be given in analytical form.

${ }^{1}$ A. A. Ukhanov, A. Stintz, P. G. Eliseev, and K. J. Malloy, Appl. Phys. Lett. 84, 1058 (2004).

${ }^{2}$ T. Akiyama, N. Hatori, Y. Nakata, H. Ebe, and M. Sugawara, Electron. Lett. 38, 1139 (2002).

${ }^{3}$ A. V. Uskov, E. P. O'Reilly, D. McPeake, N. N. Ledentsov, D. Bimberg, and G. Huyet, Appl. Phys. Lett. 84, 272 (2004).

${ }^{4}$ Z. Yuan, B. E. Kardynal, R. M. Stevenson, A. J. Shields, C. J. Lobo, K. Cooper, N. S. Beattie, D. A. Ritchie, and M. Pepper, Science 295, 102 (2002).

${ }^{5}$ G. Bester, A. Zunger, and J. Shumway, Phys. Rev. B 71, 075325 (2005).

${ }^{6}$ E. C. Le Ru, P. Howe, R. Murray, and T. S. Jones, Phys. Rev. B 67, 165303 (2003).

${ }^{7}$ J. M. Moison, C. Guille, F. Houzay, F. Barthe, and M. Van Rompay, Phys. Rev. B 40, 6149 (1989).

${ }^{8}$ J. M. García, J. P. Silveira, and F. Briones, Appl. Phys. Lett. 77, 409 (2000).

${ }^{9}$ P. B. Joyce, T. J. Krzyzewski, G. R. Bell, B. A. Joyce, and T. S. Jones, Phys. Rev. B 58(24), R15981-R15984 (1998).

${ }^{10}$ B. Lita, R. S. Goldman, J. D. Phillips, and P. K. Bhattacharya, Surf. Rev. Lett. 7, 539 (2000).

${ }^{11}$ A. Rosenauer, D. Gerthsen, D. Van Dyck, M. Arzberger, G. Boehm, and G. Abstreiter, Phys. Rev. B 64, 245334 (2001).

${ }^{12}$ Q. Xie, A. Madhukar, P. Chen, and N. P. Kobayashi, Phys. Rev. Lett. 75, 2542 (1995).
${ }^{13}$ M. O. Lipinski, H. Schuler, O. G. Schmidt, K. Eberl, and N. Y. Jin-Phillip, Appl. Phys. Lett. 77, 1789 (2000).

${ }^{14}$ K. Eberl, M. O. Lipinski, Y. M. Manz, W. Winter, N. Y. Jin-Phillipp, and O. G. Schmidt, Physica E (Amsterdam) 9, 164 (2001).

${ }^{15} \mathrm{H}$. Heidemeyer, S. Kiravittaya, C. Mueller, N. Y. Jin-Phillipp, and O. Schmidt, Appl. Phys. Lett. 80, 1544 (2002).

${ }^{16}$ P. Howe, E. C. Le Ru, E. Clarke, B. Abbey, R. Murray, and T. S. Jones, J. Appl. Phys. 95, 2998 (2004).

${ }^{17}$ C. H. Roh, Y. J. Park, K. M. Kim, Y. M. Park, E. K. Kim, and K. B. Shim, J. Cryst. Growth 226, 1 (2001).

${ }^{18}$ Q. Xie, P. Chen, and A. Madhukar, Appl. Phys. Lett. 65, 2051 (1994).

${ }^{19}$ P. W. Fry et al., Phys. Rev. Lett. 84, 733 (2000).

${ }^{20}$ D. M. Bruls, P. M. Koenraad, H. W. M. Salemink, J. H. Wolter, M. Hopkinson, and M. S. Skolnick, Appl. Phys. Lett. 82, 3758 (2003).

${ }^{21}$ X. Z. Liao, J. Zou, X. F. Duan, D. J. H. Cockayne, R. Leon, and C. Lobo, Phys. Rev. B 58, R4235 (1998).

${ }^{22}$ I. Kegel, T. H. Metzger, A. Lorke, J. Peisl, J. Stangl, G. Bauer, J. M. Garcia, and P. M. Petroff, Phys. Rev. Lett. 85, 1694 (2000).

${ }^{23}$ P. D. Siverns, S. Malik, G. McPherson, D. Childs, C. Roberts, R. Murray, B. A. Joyce, and H. Davock, Phys. Rev. B 58, R10127 (1998).

${ }^{24}$ P. Howe, B. Abbey, E. C. Le Ru, R. Murray, and T. S. Jones, Thin Solid Films 464-465, 225 (2004).

${ }^{25}$ P. B. Joyce, T. J. Krzyzewski, P. H. Steans, G. R. Bell, J. H. Neave, and T. S. Jones, J. Cryst. Growth 244, 39 (2002).

${ }^{26}$ B. Rahmati, W. Jäger, H. Trinkaus, R. Loo, L. Vescan, and H. Lüth, Appl. Phys. A: Mater. Sci. Process. 62, 575 (1996).

${ }^{27}$ B. Jogai, J. Appl. Phys. 88, 5050 (2000).

${ }^{28}$ Q. X. Pei, C. Lu, and Y. Y. Wang, J. Appl. Phys. 93, 1487 (2003).

${ }^{29}$ T. Benabbas, Y. Androussi, and A. Lefebvre, J. Appl. Phys. 86, 1945 (1999).

${ }^{30}$ G. Springholz et al., Mater. Sci. Eng., B B88, 143 (2002).

${ }^{31}$ G. S. Pearson and D. A. Faux, J. Appl. Phys. 88, 730 (2000).

${ }^{32}$ H. Shin, Y.-H. Yoo and W. Lee, J. Phys. D 36, 2612 (2003).

${ }^{33}$ W. Lee, J.-M. Myoung, Y.-H. Yoo, and H. Shin, Solid State Commun. 132, 135 (2004)

${ }^{34}$ A. D. Andreev, J. R. Downes, D. A. Faux, and E. P. O'Reilly, J. Appl. Phys. 86, 297 (1999).

${ }^{35}$ A. D. Andreev and E. P. O'Reilly, Phys. Rev. B 62, 15851 (2000).

${ }^{36}$ J. A. Barker and E. P. O'Reilly, Phys. Rev. B 61, 13840 (2000).

${ }^{37}$ J. D. Eshelby, Proc. R. Soc. London, Ser. A 241, 376 (1957).

${ }^{38}$ J. H. Davis, J. Appl. Phys. 84, 1358 (1998).

${ }^{39}$ M. De Giorgi, A. Taurino, A. Passaseo, M. Catalano, and R. Cingolani, Phys. Rev. B 63, 245302 (2001).

${ }^{40}$ E. Penev, P. Kratzer, and M. Scheffler, Phys. Rev. B 64, 085401 (2001).

${ }^{41}$ I. S. Gradshteyn and I. M. Ryzhik, Table of Integrals, Series, and Products, 6th ed., (Academic, San Diego, 2000). 